ANNA KAPUŚCIŃSKA

\title{
Sprachliche Verfremdung oder Einbürgerung beim Übersetzen von Archaismen aus der Perspektive des heutigen Rezipienten am Beispiel des Gedichts Romantyczność von Adam Mickiewicz
}

Schlüsselwörter: Literaturübersetzung, Archaismen, Verfremdung, Einbürgerung, Wirkungsäquivalenz

Der Begriff „Archaismus” wird in der Alltagssprache als ,einzelnes archaisches Element" (vgl. Duden 2003: 163) verstanden. Sprachwissenschaftlich sind Archaismen die aus dem Gebrauch geratenen Wortformen, Wörter, Wortbedeutungen, Phraseologismen und syntaktischen Konstruktionen (vgl. Kurkowska, Skorupka 2001: 67). Die Bezeichnung ,archaisch” aus der Duden-Definition wird von Kurkowska und Skorupka als ,aus dem Gebrauch geraten” spezifiziert und das dudensche „Element” kann nach ihnen zu unterschiedlichen Sprachebenen gehören.

Auf der phonologischen Ebene treten phonetische Archaismen auf, in denen nur ein einzelnes Phonem archaisch ist (teutsch statt deutsch). Unter morphologischen Archaismen unterscheidet man zwischen flexivischen Archaismen, die veraltete Flexionsformen aufweisen (ward statt wurde) (vgl. Holly 2001: 431; nach Fix, Gardt, Knape 2009: 1174) und Wortbildungsarchaismen, die nicht mehr gebräuchliche Wortbildungen sind (worinnen statt 
in dem) (vgl. Holly 2001: 431; nach Fix, Gardt, Knape 2009: 1174)1. Hinsichtlich der Semantik gibt es lexikalische und semantische Archaismen. Mit einem lexikalischen Archaismus hat man dann zu tun, wenn ein Lexem nicht mehr gebräuchlich ist (der Barbier statt der Frisör) und mit einem semantischen Archaismus, wenn sich die Bedeutung eines Lexems verändert hat (die Hochzeit $^{2}$ ). Schließlich gehören zur syntaktischen Ebene phraseologische Archaismen, d. h. die Phraseologismen, die zwar verständlich sind, aber deren Ursprung unklar ist (jmdm. etw. in die Schuhe schieben (vgl. Duden 2002: 684) in der Bedeutung ,jmdm. die Schuld an etw. zuschieben”) und syntaktische Archaismen, die eine gegenwärtig ungewöhnliche Verbindung von mindestens zwei Wörtern sind (,kapriziöse Nominalisierungen mit Linkseinbettungen (nach endlich bewirkter Zahlung, bei einer brauchtümlichen Weinfestlichkeit), veraltete Präpositionsfügungen (für altmodisch gegolten) und vorangestellte Genitivattribute (des Konfuzius Erinnerung)") (vgl. Holly 2001: 431; nach Fix, Gardt, Knape 2009: 1174)3.

Der Archaismus wird meistens als ein bewusstes Stilmittel verstanden (vgl. Schweikle 1990: 25; Kurkowska, Skorupka 2001: 67). Als archaisiert gelten daher die Texte, die in einer Sprachvariante auftreten, die nicht nur für ihren Rezipienten, sondern auch für ihren Emittenten veraltet ist. Die Archaisierung in diesem Sinne ist mit der für den heutigen Rezipienten veralteten Sprachvariante älterer Texte nicht zu verwechseln (vgl. Schreiber 1993: 77, Anm. 156).

Während eine solche Sprachvariante keine Archaisierung aus der Emittentenperspektive ist, wird sie von dem heutigen Rezipienten genauso wie eine Archaisierung wahrgenommen. Auch in diesem Falle erweist sich die von Kurkowska und Skorupka vorgeschlagene Klassifizierung als anwendbar. Die veralteten Sprachelemente älterer Texte unterscheiden sich sprach-

1 Holly macht keine Unterscheidung zwischen flexivischen Archaismen und Wortbildungsarchaismen. Deswegen werden die Lexeme ward und worinnen als Beispiele für morphologische Archaismen angeführt (vgl. Holly 2001: 431; nach Fix, Gardt, Knape 2009: 1174).

2 Das Lexem die Hochzeit stand im Mittelhochdeutschen für, jedes große kirchliche und weltliche Fest' (Duden 2001: 62) und gegenwärtig steht es nur für das Fest der Vermählung.

${ }^{3}$ Die angeführten Beispiele für die einzelnen Typen von Archaismen sind nicht dem Werk von Kurkowska und Skorupka entnommen, weil sich die Autoren mit Archaismen nur am Beispiel der polnischen Sprache befassen. 
lich nicht von den Archaismen aus der Emittentenperspektive. Aus diesem Grund werden sie in dem vorliegenden Beitrag als Archaismen aus der Perspektive des heutigen Rezipienten (Rezipienten-Archaismen) bezeichnet.

Weil die Wiedergabe der Archaismen in der Übersetzung eines Textes weniger umstritten ist, beschränkt sich dieser Beitrag auf das Problem der Übertragung von Rezipienten-Archaismen, d. h. der sprachlichen ${ }^{4}$ Historisierung oder Modernisierung (vgl. Schreiber 1993: 77) älterer Texte.

Bereits Schleiermacher meint: „Entweder der Übersetzer läßt den Schriftsteller möglichst in Ruhe, und bewegt den Leser ihm entgegen; oder er läßt den Leser möglichst in Ruhe, und bewegt den Schriftsteller ihm entgegen." (Schleiermacher 1838: 218, zit. nach Apel, Kopetzki 2003: 89). Den Leser dem Schriftsteller entgegen zu bewegen, bedeutet hier die für den Rezipienten veraltete Sprachvariante in der Zielsprache mit Archaismen als veraltet wiederzugeben. Den Schriftsteller dem Leser entgegen zu bewegen, heißt die für den Emittenten gegenwärtige Sprachvariante durch die für den Rezipienten gegenwärtige zu ersetzen. Anders gesagt können solche Texte entweder verfremdend oder einbürgernd übersetzt werden (vgl. Schreiber 1993: 73).

Um das Problem der sprachlichen Verfremdung und Einbürgerung älterer Texte an einem Beispiel zu veranschaulichen, werden unten vier letzte Strophen $^{5}$ eines der Programmgedichte von Adam Mickiewicz Romantyczność (Romantik) (1821) im Original und in der deutschen Übersetzung von Karl Dedecius einer kontrastiven Analyse unterzogen. Die Strophen werden aus dem Text im Hinblick auf die Anzahl der Rezipienten-Archaismen exzerpiert. Damit die Modernisierung des Textes sichtbarer ist, werden die RezipientenArchaismen im ausgangssprachlichen (AS) Text und die ihnen mindestens teilweise semantisch entsprechenden Wörter bzw. Wortgruppe im zielsprachlichen (ZS) Text (falls sie vorhanden sind) unterstrichen.

${ }^{4}$ Neben der sprachlichen nennt Schreiber auch die kulturelle Verfremdung bzw. Einbürgerung (vgl. Schreiber 1993: 76). Die kulturelle Verfremdung kommt eher selten vor und wird meistens nicht als eine Übersetzung, sondern eine Adaptation betrachtet (vgl. Kelletat 1987: 46, nach Schreiber 1993: 78).

${ }^{5}$ Diesen vier Strophen entsprechen fünf Strophen in der deutschen Übersetzung. Es ist jedoch nicht auszuschließen, dass die Leerzeile zwischen dem zweiten und dritten Vers des angeführten Textes ein Druckfehler ist. 
ROMANTYCZNOŚĆ

I ja to słyszę, i ja tak wierzę,

Płaczę i mówię pacierze.

«Słuchaj dzieweczko!» - krzyknie śród zgiełku Starzec i na lud zawoła:

«Ufajcie memu oku i szkiełku,

Nic tu nie widzę dokoła.

Duchy karczemnej tworem gawiedzi, w głupstwa wywarzone kuźni.

Dziewczyna duby smalone bredzi, A gmin rozumowi bluźni».

\section{ROMANTIK}

Ich sehe, ich glaub es auch

Und bete nach altem Brauch.

»So höre! « ruft aus der Menge Ein Greis, »Vertrau meinem Glas Und Aug, ich seh nur Gedränge, Sonst nichts nach menschlichem Maß.

Die Geister sind Schnapsideen Der Saufbrüder hierzulande. Das Mädchen glaubt, was zu sehen, Sie faselt wider Verstand.«
«Dziewczyna czuje - odpowiadam skromnie A gawiedź wierzy głęboko; Czucie i wiara silniej mówi do mnie Niż mędrca szkiełko i oko.

Martwe znasz prawdy, nieznane dla ludu, Widzisz świat w proszku, w każdej gwiazd iskierce;

Nie znasz prawd żywych, nie obaczysz cudu! Miej serce i patrzaj w serce!»

(Mickiewicz 1972: 63)
»Sie glaubt an die Wiederkehr; Ihr Glaube ist keine Grille; Gefühl und Glaube sind mehr Als des Klüglers Auge und Brille.

Du krankst, das Volk ist gesunder, Dein All ist Staub, statt Schmerzen; Du kennst nicht das wahre Wunder! Hab Herz und schau in die Herzen!« (Mickiewicz 1994: 59)

Der angeführte AS-Text enthält sechzehn Rezipienten-Archaismen, darunter einen flexivischen Rezipienten-Archaismus (patrzaj), einen Wortbildungs-Rezipienten-Archaismus (dzieweczko), neun lexikalische (śród, memu, dokoła, karczemnej, gawiedzi, duby smalone, gmin, gawiedź und obaczysz), drei semantische (wywarzone, czucie, mówi) und zwei syntaktische Rezipienten-Archaismen (mówię pacierze, rozumowi bluźni). Von den RezipientenArchaismen in dem AS-Text werden keine in dem ZS-Text wiedergegeben. Die nachfolgende Analyse der Rezipienten-Archaismen beschänkt sich auf die Elemente, die im ZS-Text direkt übertragen sind. Damit wird es besonders deutlich gezeigt, dass der Übersetzer jeweils das gegenwärtige Äquivalent wählt und sich damit der gängigen Sprachvariante anpasst. 
Der erste Rezipienten-Archaismus gehört der syntaktischen Sprachebene. Das ist die Wortgruppe mówię pacierze. Zwar ist die pluralische Form des Substantivs pacierz möglich (vgl. Sobol 1995: 591), aber in diesem Kontext wirkt sie eher veraltend. Von den beiden angegebenen Bedeutungen ('zbiór modlitw' und 'modlitwa') scheint die zweite für Pluralisierung tauglicher zu sein. Gegenwärtig verwendet man das Substantiv eher nicht als ein Gebet, sondern als eine Abfolge von Gebeten, d. h. in der ersten Bedeutung. So wird pacierz selten pluralisiert. Im ZS-Text wird die Wortgruppe mit der gegenwärtigen Verbform bete wiedergegeben.

Eine veraltende Form eines Personalpronomens (тети statt mојети) ist ein lexikalischer Rezipienten-Archaismus. Er wird mit der gegenwärtigen Form meinem wiedergegeben.

Die Wortgruppe karczemnej [...] gawiedzi besteht aus zwei lexikalischen Rezipienten-Archaismen. Der erste ist ein vom Substantiv karczma abgeleitetes Adjektiv. Es ist nicht nur aus sprachlichen, sondern auch aus kulturellen Gründen veraltet, weil sein Denotat, eine ehemalige Art einer polnischen Dorfgaststätte, außer in Eigennamen ${ }^{6}$, nicht mehr existiert ${ }^{7}$. Der zweite Rezipienten-Archaismus ist dagegen vor allem sprachlich bedingt. Gawiedź ist eine veraltete Bezeichnung für einfaches Volk. Dedecius gibt die ganze Wortgruppe mit einem gegenwärtigen Äquivalent Saufbrüder wieder.

Der nächste Rezipienten-Archaismus rozumowi bluźni ist mit syntaktischen Kategorien zu beschreiben. Das Verb bluźnić tritt in der übertragenen, heute aber immer noch gebräuchlichen Bedeutung auf (vgl. Sobol 1995: 55). Dabei ist jedoch eine qualitative Verschiebung im Kasussystem in Bezug auf den gegenwärtigen Gebrauch zu beobachten. Das Verb verbindet sich heute mit der Präposition przeciw oder przeciwko und nicht, wie im AS-Text, mit einem Substantiv im Dativ. Der Übersetzer entscheidet sich an dieser Stelle für die Wortgruppe faselt wider Verstand, wobei er die übliche gegenwärtige Rektion des Verbs faseln behält.

Das Substantiv czucie ist in dem Gedicht ein semantischer RezipientenArchaismus. Zwar ist dieses Lexem immer noch gebräuchlich, aber eher

${ }^{6}$ Das Lexem karczma tritt gegenwärtig nur noch in den Namen von manchen Restaurants auf.

7 In Bezug auf Bezeichnungen für nicht mehr existente Denotate sprechen Kurkowska und Skorupka von Sacharchaismen, die in ihrer Archaismen-Klassifizierung nicht erfasst sind (vgl. Kurkowska, Skorupka 2001: 70). 
im Sinne des Tastsinns. Für das Substantiv Gefühl, das im Gedicht vermutlich gemeint wird und das in dem AS-Text wiedergegeben wird, steht in der gegenwärtigen Variante der polnischen Sprache ein anderes Substantiv-uczucie.

Der letzte im angeführten AS-Text enthaltene Rezipienten-Archaismus, der im ZS-Text ein Äquivalent findet, ist das flexivische Element patrzaj als Imperativform vom Verb patrzeć zu verstehen. Die gegenwärtige Form davon lautet patrz. In dem ZS-Text wird das mit der gegenwärtigen Imperativform schau wiedergegeben.

Rezipienten-Archaismen im analysierten AS-Text werden im ZS-Text kein einziges Mal mit einem Archaismus wiedergegeben. Ähnlich verfährt Dedecius mit Rezipienten-Archaismen in einem anderen Gedicht von Mickiewicz - Oda do młodości (Ode an die Jugend), das der Gegenstand der Analyse von Chojnowski ist (vgl. Chojnowski 2005: 266-268).

In seiner theoretischen Studie zu Fragen der Übersetzungskunst drückt Dedecius seine Überzeugung aus, dass man nur das übersetzen soll, was man in dem ausgangssprachlichen Text selbst entdeckt, was entweder vorbildlich oder erfinderisch hinsichtlich der künstlerischen Form, oder wichtig und notwendig für den Inhalt ist (vgl. Dedecius 1988: 68). Die Tatsache, dass er die veraltete Sprachvariante dieses Textes nicht für das Vorbildliche, Erfinderische und daher zu Rekonstruierende hält, ist vermutlich damit zu erklären, dass ,die Wirkung der Übersetzung und nicht die stilistische Treue dem Original gegenüber bei ihm im Vordergrund steht" (Chojnowski 2005: 268).

Die Wirkungsäquivalenz steht auch bei Keller im Vordergrund. „Übersetzen heißt demnach versuchen, genau die Wirkungen, die ein Autor mit dem zu übersetzenden Text zu erzielen intendiert, mit einem Text einer anderen Sprache zu erzielen.” (Keller 1997: 12). Ob es überhaupt möglich ist, dieselbe Wirkung zu erzielen, ist umstritten. Bei Kloepfer wird die Übersetzung ,zu einem konstitutiven Bestandteil der Wirkungsgeschichte" (Apel, Kopetzki 2003: 41) eines Textes. Mit dieser Definition wird darauf hingewiesen, dass die Wirkung eines Textes dynamisch ist. Selbstverständlich wirkt beispielsweise die Übersetzung eines appellativen Textes aus dem 19. Jh., der zu einem Aufstand aufruft, nicht mehr appellativ, sondern informativ als ein historischer Quellentext. Unabhängig davon, ob er sprachlich einbürgernd oder verfremdend übersetzt wurde, darf man nicht vergessen, dass sich der außensprachliche Kontext verändert hat. In solchen Fällen ist die gewisse Diskre- 
panz zwischen der Textfunktion und Textwirkung zu beobachten (vgl. Große 1976: 68-69; nach Brinker 2010: 88-89). Obwohl die appellative Funktion des Textes erkennbar bleibt, ist es unmöglich, die appellative Wirkung eines solchen Textes wiederherzustellen.

Nach der Konzeption des sprachlichen Weltbildes von Sapir und Whorf ist die totale Äquivalenz - also auch Wirkungsäquivalenz - sogar beim Übersetzen gegenwärtiger Texte nicht erreichbar. Die Menschen, die sehr verschiedene Grammatiken ${ }^{8}$ benützen, gelangen nämlich zu verschiedenen Ansichten von der Welt (vgl. Whorf 1963: 20-21; nach Stolze 2005: 30). Die SapirWhorf-Hypothese wird häufig u. a. mit dem Boas'schen Beispiel der Vielzahl von Schneebezeichnungen in der Eskimo-Sprache illustriert (vgl. Lehmann 1998: 18), das veranschaulichen lässt, wie die Realität durch die Sprache klassifiziert und widergespiegelt wird 9 .

Sapir und Whorf stützten sich in ihren Untersuchungen auf die Unterschiede zwischen der englischen Sprache, als einem Hauptbeispiel der SAESprachen (Standard Average European) und der Sprache von Hopi-Indianern (vgl. Stolze 2005: 30). Unbestreitbar unterscheiden sich die Sprachsysteme und dadurch auch die sprachlichen Weltbilder in demselben Kulturraum nicht so stark voneinander, aber auch unter ihnen sind Unterschiede besonders im Bereich der Semantik nachzuweisen. In seinen Untersuchungen zu semantischen Wortfeldern kommt Weisgerber zu der Schlussfolgerung, dass jeder Mensch in die Welt, in der er lebt, eingebettet ist und von ihr beeinflusst wird (vgl. Cieszkowski 2001: 49). In Anlehnung daran lässt sich feststellen, dass keine zwei Sprachen ein identisches Weltbild schaffen. Dasselbe gilt für zwei unterschiedliche Varianten derselben Sprache.

Besonders stark ausgeprägt sind die Unterschiede zwischen einer veralteten Variante der Ausgangsprache und einer gegenwärtigen Variante der Zielsprache. Selbstverständlich muss man der Tatsache die Rechnung tragen, dass ,nicht nur Übersetzungen, sondern auch Originale altern” (Kloepfer 1967: 82; zit. nach Schreiber 1993: 77) und dass sich die Wirkung eines

${ }^{8}$ Mit dem Begriff „Grammatiken” werden linguistische Systeme gemeint (vgl. Kunczik, Zipfel 2005: 36).

${ }^{9}$ Mit der Tatsache, dass z. B. die Engländer nur eine Bezeichnung für den Schnee haben, wird von Boas nicht bewiesen, dass sie die Unterschiede zwischen verschiedenen Schneesorten nicht sehen können. Inzwischen wird dieses Beispiel manchmal als Beweis für sprachlichen Determinismus interpretiert und als solcher kritisiert (vgl. Lehmann 1998: 106). 
Textes auch innerhalb einer Sprache verändern kann. Demnach muss man ein veraltetes Original zuerst intralingual ${ }^{10}$ und erst danach interlingual übersetzen (vgl. Jakobson 1974: 155; nach Apel, Kopetzki 2003: 5).

Die intralinguale Übersetzung solcher Texte gehört nach der Klassifizierung von Wills zur Verstehensphase ${ }^{11}$, ,in der der Übersetzer den ausgangssprachlichen Text auf seine Sinn- und Stilintention hin analysiert" (Wills 1977: 72; zit. nach Apel, Kopetzki 2003: 5). Die Analyse der Sinnintention kann nur durch intralinguale Übersetzung in die gegenwärtige Sprachvariante erfolgen. In der Verstehensphase wird also der Text immer eingebürgert, auch wenn er dann wieder verfremdend als archaisiert wiedergegeben wird. In Anlehnung daran bedeutet eine verfremdende Übersetzung, dass der Übersetzer (in dem Beitrag ,der sekundäre Emittent” genannt, im Unterschied zum ,primären Emittenten” - dem Autor) einen bereits entdeckten Sinn vor dem Rezipienten wieder versteckt, um ihn dazu zu bewegen, bei der Textrezeption selbst aus der veralteten in die gegenwärtige Sprachvariante intralingual zu übersetzen. Das scheint nur dann zweckmäßig zu sein, wenn der primäre Emittent die zeitgenössischen Rezipienten auch zu einer solchen Handlung zwingt.

Auch wenn eine totale Wirkungsäquivalenz unerreichbar ist, soll man die Wirkung eines Textes nicht zusätzlich verunstalten. Im Falle eines älteren Textes wie das Gedicht von Mickiewicz hätte eine verfremdende Übersetzung eine solche Folge gehabt. Obwohl der Text von dem gegenwärtigen Rezipienten als archaisiert wahrgenommen wird, war die Archaisierung keine Absicht des primären Emittenten. Die Übersetzung eines älteren Textes in die veraltete Sprachvariante würde auf den gegenwärtigen Rezipienten einem unbeabsichtigten Eindruck entweder der Feierlichkeit oder der Ironie machen (vgl. Schweikle 1990: 25; Lewandowski 1990: 95; Bußmann 1990: 83; Kurkowska, Skorupka 2001: 70) und dadurch die Wirkung des Textes verändern.

10 Koller unterscheidet zwischen der „Anpassung”, die nur die Ebene der Phoneme oder Grapheme betrifft, und der intralingualen Übersetzung, wo auch andere Ebenen des Textes verändert werden (vgl. Koller 1992: 83-84; nach Schreiber 1993: 25). Weil sich der vorliegende Beitrag nicht nur mit der Verfremdung, bzw. Einbürgerung der Orthographie befasst, ist es berechtigt, den Begriff, ,intralinguale Übersetzung” zu verwenden.

11 Neben der Verstehensphase unterscheidet Wills auch die Rekonstruktionsphase (vgl. Wills 1977: 72; nach Apel, Kopetzki 2003: 5). 
Nord spricht dabei von der „Loyalität” dem Sender und dem Empfänger gegenüber (vgl. Apel, Kopetzki 2003: 35). Eine verfremdende Übersetzung gilt als unloyal und wird manchmal sogar als ein Betrug angesehen. Ein solches Übersetzen ist „weder dem Originaltext treu, den es negieren muß, noch dem Rezipienten, den es betrügt" (Forget 1987: 516; zit. nach Schreiber 1993: 74).

Die oben angeführte Behauptung von Forget lässt mühelos nachvollziehen, inwiefern die kommunikativen Ansätze zur Übersetzungswissenschaft den Begriff der Treue revolutioniert haben. Eine Übersetzung, die im traditionellen Sinne treu ist und von Schleiermacher als die einzige Möglichkeit, den „Geist der Sprache” (vgl. Stolze 2003: 145) zu behalten, angezeigt wird, hält man heutzutage für einen Betrug oder eine Fälschung ${ }^{12}$.

Kennzeichnend ist, dass die Loyalität bei Nord nicht zwei Texte, sondern zwei Parteien der Kommunikation betrifft. Demnach beschäftigt man sich nicht mit Übersetzungen als Texten, sondern viel mehr mit dem Übersetzen als einer Art Kommunikation. In der Praxis geht es nicht mehr darum, die Texte äquivalent zu übertragen, sondern mit der Zielkultur erfolgreich zu kommunizieren, ,indem sie [die Übersetzung] das ausgangskulturelle Wirkungspotenzial des Textes den Leseerwartungen des Zielpublikums anpasst" (Apel, Kopetzki 2003: 35).

Anstelle der Äquivalenz im traditionellen Sinne tritt die kommunikative Äquivalenz, auch Adäquatheit genannt. Sie wird als ,die Relation zwischen einem Text und seinem, Umfeld', also Autor, Leser, Produktions- und Rezeptionsbedingungen usw." (Albrecht 1998: 76) definiert. Danach ist die Aufgabe der Übersetzung, diese Relation wiederherzustellen. Weil der AS-Text des Gedichts Romantyczność für Mickiewicz als den primären Emittenten und für seine zeitgenössischen Rezipienten gegenwärtig war, als solcher produziert und rezipiert wurde, soll dasselbe für den ZS-Text gelten. Die einbürgernde Übersetzung von Dedecius erfüllt diese Forderung, indem sie für den sekundären Emittenten sowie für seine Rezipienten gegenwärtig ist, als gegenwärtig produziert und rezipiert wird.

12 Wittkop bezeichnet die Übersetzer, die verfremdend übersetzen als „Fälscher“, weil sie die Sprache nachahmen, in der sie nicht wirklich reden (vgl. Wittkop 1995: 440). 


\section{Literaturverzeichnis}

Albrecht J., 1998, Literarische Übersetzung. Geschichte, Theorie, kulturelle Wirkung, Darmstadt: Wissenschaftliche Buchgesellschaft.

Apel F., Kopetzki A., 2003, Literarische Übersetzung, Stuttgart-Weimar: Verlag J. B. Metzler.

BrINKER K., 2010, Linguistische Textanalyse. Eine Einführung in Grundbegriffe und Methoden, Berlin: Erich Schmidt Verlag.

Bussmann H., 1990, Lexikon der Sprachwissenschaft, Stuttgart: Alfred Kröner Verlag. Chojnowski P., 2005, Zur Strategie und Poetik des Übersetzens, Berlin: Frank \& Timme.

Cieszkowski M., 2001, Zeichen und Zeichenrelationen. Beispielhaft dargestellt an Tier-Textemen aus Friedrich Nietzsches Werk „Also sprach Zarathustra”, Bydgoszcz: Wydawnictwo Uczelniane Akademii Bydgoskiej im. Kazimierza Wielkiego.

Dedecius K., 1988, Notatnik tlumacza, Warszawa: Czytelnik.

Duden, 2001, Das Herkunfstwörterbuch, Bd. 7, Mannheim-Leipzig-Wien-Zürich: Dudenverlag.

Duden, 2002, Redewendungen, Bd. 11, Mannheim-Leipzig-Wien-Zürich: Dudenverlag.

Duden, 2003, Deutsches Universalwörterbuch, Mannheim-Leipzig-Wien-Zürich: Dudenverlag.

Fix U., Gardt A., Knape J. (Hg.), 2009, Rhetorik uns Stilistik. Ein internationales Handbuch historischer und systematischer Forschung. Band 2, Berlin: Monton de Gruyter.

FoRget P., 1987, Aneignung oder Annexion. Übersetzen als Modellfall textbezogener Interkulturalität, in: A. Wierlacher (Hg.), Perspektiven und Verfahren interkultureller Germanistik, München: Judicium, S. 511-513.

Grosse E. U., 1976, Text und Kommunikation. Eine linguistische Einführung in die Funktionen der Texte, Stuttgart: Kohlhammer.

Holly W., 2001, „Gehobener Stil” als sozialer Stil. „Das neue Notizbuch” von Johannes Gross als Textbeispiel, in: E.-M. Jakobs, A. Rothkegel (Hg.), Perspektiven auf Stil, Tübingen: Niemeyer, S. 423-441.

JAKobson R., 1974, Linguistische Aspekte der Übersetzung, in: R. Jakobson, Form und Sinn. Sprachwissenschaftliche Betrachtungen, München: Wilhelm Fink Verlag, S. 154-161.

Keller R. (Hg.), 1997, Linguistik und Literaturübersetzen, Tübingen: Narr.

Kelletat A. F., 1984, Herder und die Weltliteratur. Zur Geschichte des Übersetzens im 18. Jahrhundert, Frankfurt a. M.: Peter Lang. 
KLOEPFER R., 1967, Theorie der literarischen Übersetzung, München: Wilhelm Fink.

Koller W., 1992, Einführung in die Übersetzungswissenschaft, Heidelberg-Wiesbaden: Quelle \& Meyer.

KuncziK M., Zipfel A., 2005, Publizistik: Ein Studienbuch, Stuttgart: UTB.

Kurkowska H., Skorupka S., 2001, Stylistyka polska. Zarys, Warszawa: Wydawnictwo Naukowe PWN.

LeHMANN B., 1998, ROT ist nicht »rot« und nicht [rot]. Eine Bilanz und Neuinterpretation der linguistischen Relativitätstheorie, Tübingen: Gunter Narr Verlag.

LewANDOWsKi T., 1990, Linguistisches Wörterbuch 1, Heidelberg-Wiesbaden: Quelle \& Meyer.

Mickiewicz A., 1972, Wiersze, Warszawa: Spółdzielnia Wydawnicza „Czytelnik”.

Mickiewicz A., 1994, Dichtung und Prosa: ein Lesebuch, Übers. von K. Dedecius, Frankfurt a. M.: Suhrkamp.

Schleiermacher F., 1838, Ueber die verschiedenen Methoden des Uebersetzens, Berlin: Reimer.

SCHREIBER M., 1993, Übersetzung und Bearbeitung: Zur Differenzierung und Abgrenzung des Übersetzungsbegriffs, Tübingen: Gunter Narr Verlag.

SchweIkle G. I. (Hg.), 1990, Metzler Literatur Lexikon. Begriffe und Definitionen, Stuttgart: J. B. Metzlersche Verlagsbuchhandlung.

Sobol E. (Hg.), 1995, Mały słownik języka polskiego, Warszawa: Wydawnictwo Naukowe PWN.

Stolze R., 2003, Hermeneutik und Translation, Tübingen: Gunter Narr Verlag.

Stolze R., 2005, Übersetzungstheorien: Eine Einleitung, Tübingen: Gunter Narr Verlag.

Whorf B. L., 1963, Sprache, Denken, Wirklichkeit. Beiträge zur Metalinguistik und Sprachphilosophie, Reinbek b. Hamburg: Rowohlt-Taschenbuch-Verlag.

Wills W., 1977, Übersetzungswissenschaft. Probleme und Methoden, Stuttgart: Klett.

Wiтtкор G., 1995, Die Dichter und ihr Fälscher. Notizen aus der Unterwelt, Sinn und Form 1995/3, S. 439-443.

\section{Językowa egzotyzacja czy adaptacja w tłumaczeniu archaizmów z perspektywy dzisiejszego odbiorcy (na przykładzie wiersza Romantyczność Adama Mickiewicza)}

( streszczenie)

Niniejszy artykuł jest poświęcony zagadnieniu przekładu jednostek leksykalnych, które są archaiczne z punktu widzenia dzisiejszego odbiorcy, występujących 
w starszych tekstach literackich. Materiał badawczy został wyekscerpowany z wiersza Adama Mickiewicza Romantyczność w oryginale oraz we współczesnym tłumaczeniu Karla Dedeciusa na język niemiecki. Analiza porównawcza archaicznych jednostek leksykalnych w tekście wyjściowym oraz jednostek odpowiadających im pod względem semantycznym w tekście docelowym wykazała, iż thumacz nie zastosował archaizacji w żadnym z analizowanych przypadków. Tłumaczenie jest współczesne z perspektywy tłumacza i dzisiejszego odbiorcy, tak samo jak oryginał jest współczesny z perspektywy autora i odbiorcy w XIX w. Przeprowadzona analiza obrazuje zmiany, jakie dokonały się $\mathrm{w}$ translatoryce wraz $\mathrm{z}$ rozpowszechnieniem podejścia komunikacyjnego. Językowa adaptacja wydaje się naturalną konsekwencją faktu, iż na pierwszym miejscu zaczęto stawiać nie tekst, lecz jego odbiorcę. 